HELMINTHOLOGIA, 57, 4: 293 - 305, 2020

\title{
The neglected cestode infection: Epidemiology of Hymenolepis nana infection among children in rural Yemen
}

\author{
H. M. AL-MEKHLAFI $\left.\right|^{1,2,3}$ \\ 'Medical Research Center, Jazan University, Jazan, Kingdom of Saudi Arabia, E-mail: halmekhlafi@yahoo.com; \\ ${ }^{2}$ Department of Parasitology, Faculty of Medicine, University of Malaya, 50603 Kuala Lumpur, Malaysia; \\ ${ }^{3}$ Department of Parasitology, Faculty of Medicine and Health Sciences, Sana'a University, Sana'a, Yemen
}

Article info

Received March 12, 2020

Accepted May 26, 2020

\section{Summary}

Hymenolepis nana is the most common cestode reported in humans worldwide. It is prevalent among children in the tropics and subtropics, particularly in rural poor communities where sanitation is inadequate or lacking. This cross-sectional study aims to determine the prevalence and significant risk factors of $H$. nana infection among children in rural Yemen. Faecal samples were collected from 498 children and screened for intestinal parasites by using wet mount, formalin-ether concentration and Kato-Katz techniques. A pretested questionnaire was used to collect demographic, socioeconomic, housing condition, and personal hygiene information. Overall, $77.5 \%$ (386/498) of the children were found to be infected by at least one intestinal parasite species. The overall prevalence of $H$. nana was $17.5 \%$ (87/498). Multivariate analysis confirmed that an age of $<6$ years (adjusted odds ratio $[A O R]=4.28 ; 95 \%$ (confidence interval $[\mathrm{Cl}]=2.04,8.98$ ), presence of other family members infected with $\mathrm{H}$. nana $(\mathrm{AOR}=2.48 ; 95 \% \mathrm{Cl}=1.45,4.24)$, living in the highlands $(\mathrm{AOR}=2.87 ; 95 \% \mathrm{Cl}=$ $1.56,5.26$ ), living in a house without improved toilet facilities ( $A O R=2.19 ; 95 \% \mathrm{Cl}=1.23,3.88$ ), not washing vegetables before consumption ( $\mathrm{AOR}=2.11 ; 95 \% \mathrm{Cl}=1.06,4.19)$, and not washing hands after defecation $(A O R=1.88 ; 95 \% \mathrm{Cl}=1.08,3.27)$ were the key factors significantly associated with $H$. nana infection among the studied children. In conclusion, $H$. nana is prevalent among children in rural Yemen, particularly among preschool-aged children. Thus, an integrated and effective programme to control intestinal parasitic infections should include preschool-aged children. Such a programme should focus on providing health education on hygienic practices, providing adequate sanitation and improved sources of drinking water, and screening and treating other infected family members.

Keywords: Hymenolepis nana; infectious diseases; neglected tropical diseases; epidemiology; children; Yemen

\section{Introduction}

The cestode Hymenolepis nana, commonly known as the dwarf tapeworm, is the most prevalent cestode in humans, infecting over 75 million people worldwide (King, 2010). Infections occurs most commonly among children in the tropics and subtropics, particu- larly in underprivileged rural communities lacking sanitation and safe drinking water, with a prevalence as high as $55 \%$ (Macnish, 2001; Thompson, 2015). Hymenolepis nana is known to infect humans through ingestion of eggs in food or water contaminated with faeces from infected humans or rodents. It can also be acquired by accidental ingestion of arthropods (usually flour beetles and 
fleas that serve as intermediate hosts) containing the cysticercoid stages, which then develop into adult worms in the gastrointestinal tract (Galan-Puchades, 2015). Moreover, H. nana is the only cestode that can be transmitted directly from person to person as well as autoinfection (endogenous autoinfection) commonly occurs, a feature that may have implications in the prognosis of infection among immunocompromised patients (Lucas et al., 1979; Olson et al., 2003).

Infection with $H$. nana, called hymenolepiasis, is most often asymptomatic, but heavy infections may cause abdominal pain, diarrhoea, nausea, vomiting, loss of appetite, anal itching, and weakness (Mirdha and Samantray, 2002; Cabada et al., 2016). Moreover, headache, dizziness, sleep, and behaviour disturbances are frequently reported (O'Dempsey, 2011). Furthermore, $H$. nana infection may cause serious disseminated disease and death, particularly among immunocompromised individuals, such as AIDS patients and malnourished children (Lucas et al., 1979; Olson et al., 2003; Muehlenbachs et al., 2015; Amer et al., 2018). Nevertheless, despite several reports on $H$. nana infections among children in different countries, data on clinical relevance and morbidity caused by $H$. nana are not convincing. Currently the drug of choice for $H$. nana infection is praziquantel, administered as a single dose (Schenone, 1980; O'Dempsey, 2011).

In Yemen, a Middle Eastern country, approximately 19 million people (70 \% of the population) live below the poverty line and are food insecure (UNOCHA, 2019). Moreover, there is a severe lack of sanitation and less than $25 \%$ of the population have access to clean drinking water (Oxfam, 2015). Due to the current widespread civil war, over $55 \%$ of the country's health facilities are totally destroyed, underground water in all Yemeni cities is contaminated with sewage, and the substandard sewage treatment plants are not functioning, putting the entire population at great risk of many infectious diseases (Gavlak, 2015; Al-Mekhlafi, 2018). For instance, since 2015, there have been deadly outbreaks of cholera, dengue, leishmaniasis, and diphtheria (Al-Mekhlafi, 2018; El Bcheraoui et al., 2018; Alghazali et al., 2019).

With regards to intestinal parasites, many studies have reported on the prevalence of different intestinal parasitic infections in Yemen, including soil-transmitted helminth (STH), Giardia, Entamoeba, and Cryptosporidium infections (Azazy et al., 2002; Alyousefi et al., 2011; Al-Mekhlafi et al., 2016; Alsubaie et al., 2016; Al-Areqi et al., 2017; Al-Mekhlafi, 2017). A few of those studies have included the occurrence of $H$. nana infection in the parasitological assessment, and have reported a prevalence rate ranging between $5 \%$ and $13 \%$. However, the data on the distribution of $H$. nana infection in Yemen is limited. Moreover, data on the associated risk factors and potential impact of infection on the health and nutritional status of infected individuals is not available. Therefore, the current study was carried out among rural communities in Yemen to determine the prevalence and distribution as well as the associated risk factors of $H$. nana infection.

\section{Materials and Methods}

\section{Study design}

A community-based cross-sectional study was conducted between February and August 2016 among residents of rural communities in two governorates in Yemen. The study involved the administration of a questionnaire survey, the collection of stool and finger-prick blood samples, and a physical examination. The number of persons per household was recorded and all of the household members were invited to take part in the study. Those who agreed to participate were given unique reference codes that could be used to identify the governorates, villages, households, and individual study participants.

\section{Study area}

This study was conducted in two governorates in Yemen, namely, Taiz and Almahweet. Taiz governorate is located in the southwestern part of Yemen, $268 \mathrm{~km}$ from the capital Sana'a, at an altitude of $1,400 \mathrm{~m}$ above sea level, and has a total population of 2.9 million (NIC, 2019). The climate is hot, with relative humidity varying between $70 \%$ and $90 \%$, and a mean annual rainfall of approximately $200 \mathrm{~mm}$. There are some streams which are considered, together with many traditional dug wells, the main source of water for both drinking and domestic purposes.

Almahweet governorate, located $110 \mathrm{~km}$ northwest Sana'a at an altitude of $>2,000 \mathrm{~m}$ above sea level is categorised as a highland area, and has a total population of 0.6 million (NIC, 2019). The climate is moderate in summer and cold in winter, with relative humidity varying between $20 \%$ and $50 \%$, and a mean annual rainfall of approximately $400 \mathrm{~mm}$. There are some springs and streams in this governorate which are used to provide a supply of water for agricultural and household uses. In addition, numerous traditional small and medium-sized uncovered troughs for drinking and domestic water are located throughout the governorate. Agriculture is the main occupation of the studied population in both governorates. Cereals, vegetables, fruits and khat are the main cultivated plants in Taiz while coffee and khat are the main types of crop grown in Almahweet.

Overall, six districts (three from each governorate) were selected randomly from an official list of districts provided by the respective health office of each governorate. The selected districts were Alwazyiah, Maqbanah, and Mawaiah, in Taiz, and Alrujum, Altawilah, and Shibam-Kawkaban in Almahweet (Figure 1). Twenty-five households, the unit of sampling, were selected from each district by using a systematic random sampling technique.

\section{Sample size and study population}

After obtaining consent from the parents, all children at households were invited to participate in the study. A total of 672 children (369 boys and 303 girls) aged between 2 and 17 years were involved in the study and were given containers for stool samples. However, only 550 (81.8\%) children delivered the containers for examination 


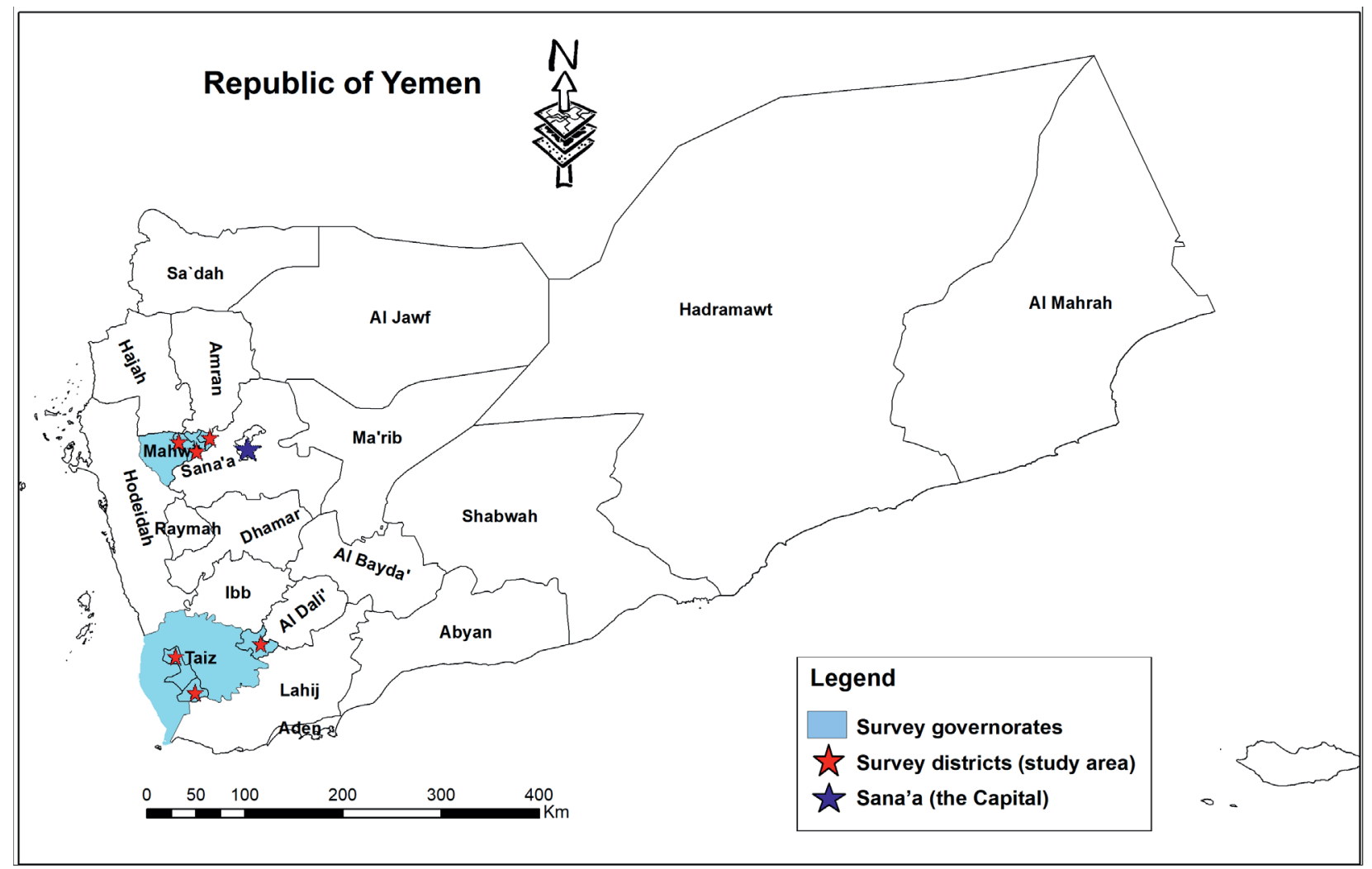

Fig. 1. A geographic map showing the districts and governorates involved in the study (6 districts within two governorates). The map was created using a licensed Esri ArcGIS 10.7 software.

while 122 (18.2 \%) failed to provide samples. A further 52 (7.7\%) children were excluded due to empty containers. Hence, a total of 498 children who had provided their faecal samples for examination and whose parents had provided complete questionnaire information were considered in the data analysis. Of these 498 participants, 256 children (169 boys and 87 girls) were from Taiz governorate and 242 children (151 boys and 91 girls) were from Almahweet governorate. This final number of participants met the estimated minimum sample size required for this study, which was calculated according to the WHO practical manual for sample size calculation (Lwanga and Lemeshow, 1991). Based on a $5 \%$ level of significance, a $95 \%$ confidence level, and design effect of 2 , the minimum sample size was 392 , assuming that the prevalence of H. nana infection in Yemen was as high as $13 \%$ as reported in a previous study (Raja'a et al., 2000).

\section{Questionnaire survey}

The children and their parents were interviewed, in their home settings, to fill out a structured pre-tested questionnaire for their family background and socioeconomic information. The questionnaire was designed in three parts. The first part centred around demographic data of the participant including age, sex, address and number of household members. The second part involved questions concerning the socioeconomic status (e.g. parents' occupational and educational status and family monthly income) and housing conditions (e.g. availability of improved toilets in the household, types of drinking water supply, and presence of domestic animals at household). The third part involved questions concerning personal hygiene (e.g. washing hands before eating, after defecation and after playing with soil, boiling drinking water before consumption, washing vegetables and fruits before consumption, indiscriminate defecation and cutting nails periodically) and clinical signs and symptoms related to intestinal parasitic infections (e.g. abdominal pain, diarrhoea, nausea and vomiting as well as a history of receiving anthelmintics treatment). The interviews were conducted by two research assistants who had received training on questionnaire administration and the purpose of the study. Moreover, housing conditions were observed and recorded by the survey team. For instance, the toilet and water supply facilities in the households were observed and classified into two types, namely, unimproved and improved, according to the WHO and UNICEF criteria (WHO/UNICEF, 2015). Accordingly, a pour flush toilet was classified as an improved toilet while a pit latrine without a slab was classified as an unimproved toilet. Similarly, piped water supplies were classified as improved water sources while other domestic water sources such as rain, wells, uncovered cistern/troughs, and streams were classified as unimproved sources. 


\section{Stool examination}

Each child was given a $100-\mathrm{mL}$ clean container with wide mouth and screw cap. Subjects were requested to provide an adequate fresh stool sample and instructed on how to avoid possible contamination in the process of collection at home. The collected containers were properly labelled, placed into proper plastic bags, kept in a cool ice box $\left(4-6^{\circ} \mathrm{C}\right)$, and transported for examination to the nearest health centre laboratory equipped with standard facilities within 5 hours of collection.

The stool samples were examined for the presence of $H$. nana and other intestinal parasites by using wet mount and formalin-ether sedimentation methods according to Cheesbrough (2005). In addition, the samples were screened by the Kato-Katz technique and egg counting was performed (WHO, 2006). The intensity of infection was recorded as number of eggs per gram (epg) of stool and classified as follows: 1-1,999: light, 2,000-9,999: moderate, and $\geq 10,000$ heavy (Chero et al., 2007; Matthys et al., 2011). A sample was considered positive if $H$. nana ova were detected by using any one of the three methods. For quality control, $10 \%$ of the stool samples were selected randomly and examined by another research assistant using the formalin-ether sedimentation technique.

\section{Statistical analysis}

Data was entered into a Windows 10 Excel spreadsheet, checked for accuracy, and then entered into the Statistical Package for the Social Sciences (SPSS) version 18 (Chicago, USA: IBM Inc.) for analysis. The dependent (H. nana infection) and independent variables (demographic, socioeconomic, housing conditions, and personal hygiene) were considered as categorical variables and presented as frequencies and percentages. The associations between variables were assessed by using Pearson's chi-square test. Odd ratios (ORs) and $95 \%$ confidence intervals (Cls) were also computed. In addition, a multiple logistic regression analysis was used to identify the significant risk factors of $H$. nana infection. In order to retain all possible risk factors, variables that showed an association with a $P$ level $\leq 0.25$ in the univariate analysis were considered in the multiple logistic regression models (Bendel and Afifi, 1977). Adjusted ORs and their corresponding $95 \%$ Cls were calculated based on the final model. The level of statistical significance was set as $P<0.05$ for all analyses.

\section{Ethical Approval and/or Informed Consent}

All procedures performed in studies involving human participants were in accordance with ethical standards of the institutional ethical committee and with the 1964 Helsinki declaration and its later amendments.

The study protocol was approved by the Medical Ethics Committee of the University of Malaya Medical Centre (Ref. No. 201411-805). Moreover, permission to commence sampling and data collection was also obtained from the respective health office of each governorate. A written and signed or thumb-printed informed consent was obtained from the parents for their participation as well as on behalf of their children.

\section{Results}

\section{General characteristics of the participants}

Four hundred and ninety-eight children aged between 2 and 17 years, with a mean age of $9.4 \pm 4.3$ years participated voluntarily in the study. Of them, 320 (64.3\%) were boys and $178(35.7 \%)$ were girls. Overall, approximately two-thirds $(62.9 \%)$ of the participants lived in households that had a low monthly income (< USD 80 ), and $43.6 \%$ of households contained an educated father (i.e., had completed at least 6 years of formal education). Poverty prevails in the study areas as evidenced by poor housing and living conditions, especially in Taiz governorate. In Taiz, most of the houses are made of stones and mud while comparatively few houses are made of bricks and concrete, whereas in Almahweet most of the houses are made of fired bricks or concrete. About a quarter $(27.7 \%)$ of the subjects lived in houses that had an improved water supply while $37.8 \%$ lived in houses with an improved toilet. The general characteristics of the participants are shown in Table 1.

\section{Prevalence and distribution of $\mathrm{H}$. nana infection}

The data on the prevalence and distribution of $H$. nana infection according to age, gender, and location is presented in Figure 2. The overall prevalence of $H$. nana infection was $17.5 \%$ (87/498). An age-dependency distribution was found, where the prevalence rate of infection decreased with age $\left(x^{2}=18.839 ; \mathrm{df}=2 ; P<\right.$ $0.001)$. The highest rate of infection was observed among preschool-aged children (<6 years) $(29.3 \%$; $39 / 133)$ while the lowest prevalence $(10.5 \%$; $15 / 143)$ was seen among those aged over 12 years. With regards to gender, there was a higher prevalence of infection among boys compared to girls (19.1\% vs $14.6 \%)$. However, this difference was not statistically significant $\left(X^{2}=1.575\right.$; df $=1, P=0.209)$. It was also found that the majority $(81.6 \% ; 71 / 87)$ of the $H$. nana infections were of light intensity while only $15.0 \%$ $(13 / 87)$ and $3.4 \%(3 / 87)$ were of moderate and heavy intensity, respectively.

With regards to the clinical presentation (Table 2), the proportion of gastrointestinal symptoms (i.e. abdominal pain, diarrhoea, vomiting, nausea, constipation, and loss of appetite) was higher among $H$. nana-infected children compared to non-infected children ( $74.7 \%$ vs $68.4 \%$ ). However, this difference was not statistically significant $\left(x^{2}=1.362 ; P=0.243\right)$. Nonetheless, the proportion of abdominal pain ( $39.1 \%$ vs $27.7 \%$; $P=0.035$ ) was significantly higher among $H$. nana-infected children than among their non-infected peers. Moreover, there was a significantly higher proportion of dizziness among $\mathrm{H}$. nana-infected children than among non-infected children (20.7 vs $11.4 \% ; P=0.020$ ). Likewise, a significant association was reported between $H$. nana infection and anal itching $(P=0.024)$. 
Table 1. General characteristics of the children participated in the study $(n=498)$.

\begin{tabular}{|c|c|}
\hline Characteristics & $\mathrm{n}(\%)$ \\
\hline \multicolumn{2}{|l|}{ Age (years) } \\
\hline$<6$ (preschool) & $133(26.7)$ \\
\hline $6-12$ & $222(44.6)$ \\
\hline$>12$ & $143(28.7)$ \\
\hline \multicolumn{2}{|l|}{ Gender } \\
\hline Boys & $320(64.3)$ \\
\hline Girls & $178(35.7)$ \\
\hline \multicolumn{2}{|l|}{ Residence (governorate) } \\
\hline Almahweet (Highland) & $242(48.6)$ \\
\hline Taiz (Coastal/foothill area) & $256(51.4)$ \\
\hline \multicolumn{2}{|l|}{ Father's occupational status } \\
\hline Not working & $80(16.1)$ \\
\hline Farmers and daily workers & $310(62.2)$ \\
\hline Government employees/professionals & $108(21.7)$ \\
\hline Father's educational level (at least primary) & $217(43.6)$ \\
\hline Low household monthly income (< USD 80) & $313(62.9)$ \\
\hline Family size ( $\leq 7$ members) & $283(56.8)$ \\
\hline Improved water supply at household & $138(27.7)$ \\
\hline Presence of improved toilet in house & $310(62.2)$ \\
\hline Presence of domestic animals at household & $321(64.5)$ \\
\hline
\end{tabular}

The children were also found to be infected with Trichuris trichiura (10.8\%), Ascaris lumbricoides (13.1\%), hookworm (12.4\%), Schistosoma mansoni (10.6\%), Enterobius vermicularis (17.9\%), Giardia duodenalis (19.1\%) and Entamoeba histolytica/dispar (24.7\%). Overall, only $28 \%$ of the $H$. nana infections were single infections while $72 \%$ were coinfections with one or more of the above-mentioned intestinal parasites. Thus, it might not be possible to elucidate the exact associations between the different infections and the gastrointestinal symptoms.

Risk factors of $\mathrm{H}$. nana infection

Potential factors associated with $H$. nana infection were analysed by univariate and multivariate analyses, the results of which are presented in Tables 3 and 4, respectively. The results in Table 3 show that the prevalence of $H$. nana infection was significantly higher among children aged below 6 years when compared with those aged over 12 years $(P<0.001)$ as well as those aged $6-12$ years $(P=0.029)$. There was also a significantly higher prevalence of $H$. nana among children living in the highlands at altitude of $>2,000 \mathrm{~m}$ above sea level (Almahweet governorate) as compared to those living in coastal/foothill areas at altitude of $1,400 \mathrm{~m}$ above sea level (Taiz governorate) $(21.9 \%$ vs $13.3 \%$; $P$ $=0.011)$. Moreover, there was a significantly higher prevalence of
$H$. nana among children whose fathers were aged $<40$ years and among children whose fathers were not working as compared to those whose fathers were aged $\geq 40$ years $(21.4 \%$ vs $13.6 \% ; P=$ 0.028 ) and those whose fathers were working as government employees/professionals ( $25.0 \%$ vs $12.0 \% ; P=0.021)$. Furthermore, the prevalence of $H$. nana was significantly higher among children from families with a low household monthly income compared to children from families with a household monthly income of $\geq$ USD $80(20.1 \%$ vs $13.0 \% ; P=0.041)$. Interestingly, there was a significantly higher prevalence of infection among children who lived with other family members infected with $H$. nana when compared to their peers in non-infected households ( $29.2 \%$ vs $12.2 \%$; $P<$ 0.001 ).

There was a significantly higher likelihood of being infected with $H$. nana among children living in a house without a toilet compared to those living in a house with toilet facilities ( $22.9 \%$ vs $14.2 \%$; $P=$ $0.013)$. With regards to personal hygiene, there was a significantly higher prevalence of infection among children who did not practise handwashing after defecation $(23.6 \%)$ or after playing with soil $(22.1 \%)$ and those who did not wash vegetables $(24.3 \%)$ or fruits $(23.3 \%)$ before consumption than among their counterparts who followed the above-mentioned hygiene practices $(P<0.05)$. 


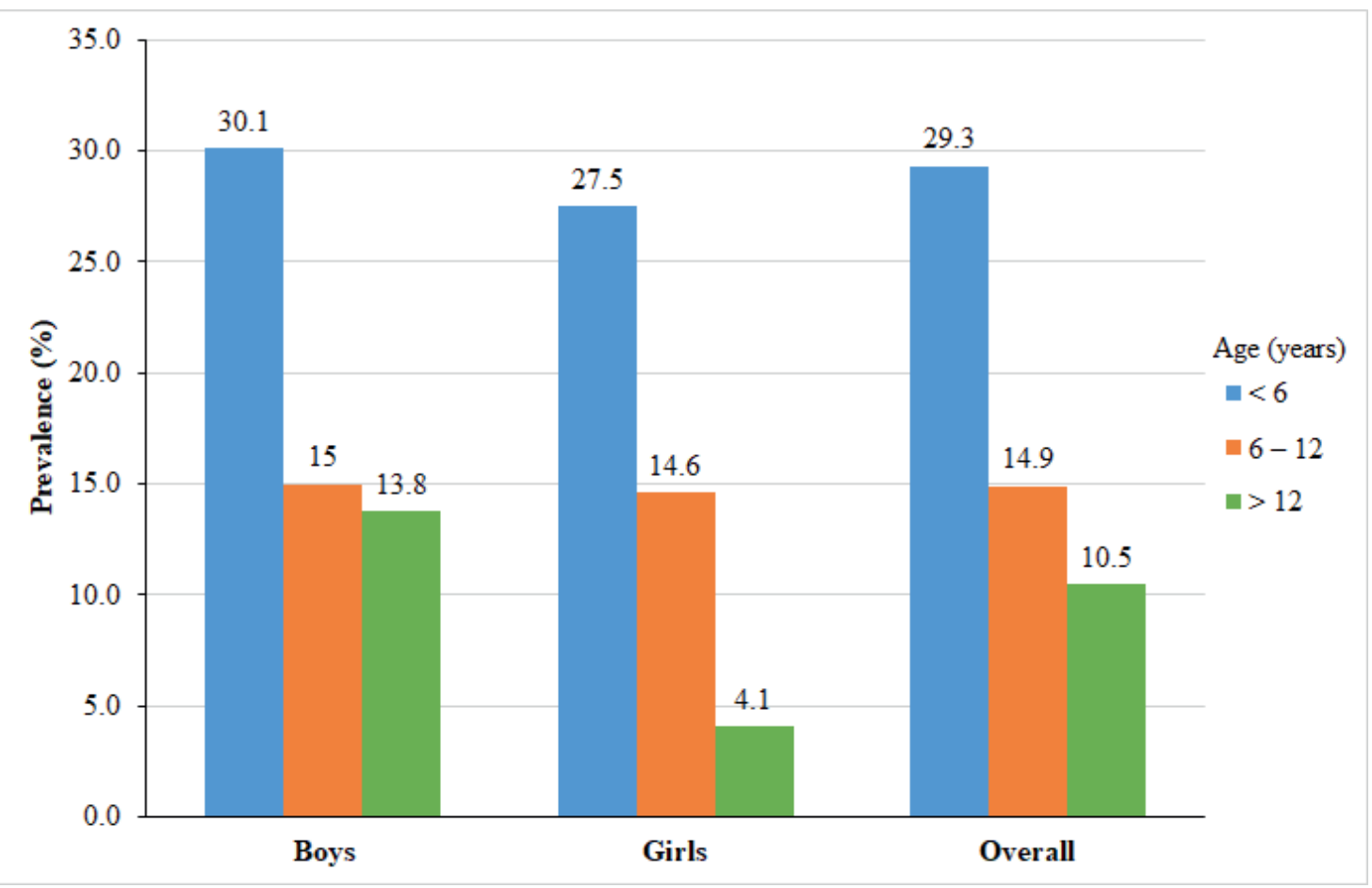

Fig. 2. Prevalence of Hymenolepis nana infection among the participants according to their age and gender $(n=498)$.

The results of the multiple logistic regression for the significant risk factors (Table 4) showed that children aged below 6 years had 4.28 times higher odds of having a $H$. nana infection when compared with older children. Moreover, children living in the highlands ( $A O R=2.87$ ), those living in a house without an improved toilet $(A O R=2.19)$, and those living with other infected family members $(A O R=2.48)$ had significantly higher odds of acquiring $H$. nana when compared to their counterparts. Similarly, children who did not wash their hands after defecation and those who did not wash vegetables before consumption had approximately two times the odds of being infected compared to their counterparts.

\section{Discussion}

The current study is the first to provide information on the epidemiology of $H$. nana in Yemen. The findings revealed a relatively high prevalence $(15.5 \%)$ of $H$. nana infection among rural children in Taiz and Almahweet governorates. Moreover, this is the highest

Table 2. Distribution of clinical signs and symptoms among Hymenolepis nana-infected children in rural Yemen $(n=498)$.

\begin{tabular}{lllll}
\hline Signs and symptoms & Overall & \multicolumn{3}{l}{ Hymenolepis nana infection } \\
\cline { 3 - 5 } & & Positive $(\mathrm{n}=87)$ & Negative $(\mathrm{n}=411)$ & $\mathrm{X}^{2}(P)$ \\
\hline Abdominal pain & 148 & $34(39.1)$ & $114(27.7)$ & $4.423(0.035)^{*}$ \\
Diarrhoea & 90 & $19(21.8)$ & $71(17.3)$ & $1.010(0.315)$ \\
Constipation & 33 & $5(5.7)$ & $28(6.8)$ & $0.132(0.717)$ \\
Nausea & 97 & $16(18.4)$ & $81(19.7)$ & $0.079(0.778)$ \\
Vomiting & 41 & $9(10.3)$ & $32(7.8)$ & $0.622(0.430)$ \\
Loss of appetite & 115 & $19(21.8)$ & $96(23.4)$ & $0.093(0.760)$ \\
Anal itching & 145 & $34(39.1)$ & $111(27.0)$ & $5.071(0.024)^{*}$ \\
Dizziness & 65 & $18(20.7)$ & $47(11.4)$ & $5.418(0.020)^{*}$ \\
Headache & 76 & $19(21.8)$ & $57(13.9)$ & $3.527(0.060)$ \\
Fatigue & 61 & $8(9.2)$ & $53(12.9)$ & $0.914(0.339)$ \\
\hline
\end{tabular}

All values are number $(\%)$.

$x^{2}$ Chi-square test statistic.

"Significant difference between the groups $(P<0.05)$. 
Table 3. Univariate analysis of factors associated with Hymenolepis nana infection among children in rural Yemen $(n=498)$.

\begin{tabular}{|c|c|c|c|c|}
\hline \multirow[t]{2}{*}{ Variables } & \multicolumn{2}{|c|}{ H. nana infection } & \multirow[b]{2}{*}{ OR $(95 \% \mathrm{Cl})$} & \multirow[b]{2}{*}{$P$ value } \\
\hline & No. examined & $\begin{array}{c}\text { Infected } \\
\mathrm{n}(\%)\end{array}$ & & \\
\hline \multicolumn{5}{|l|}{ Age (years) } \\
\hline$<6$ (preschool) & 133 & $39(29.3)$ & $3.54(1.84,6.80)$ & $<0.001^{*}$ \\
\hline $6-12$ & 222 & $33(14.9)$ & $1.49(0.78,2.86)$ & 0.227 \\
\hline$>12$ & 143 & $15(10.5)$ & 1 & \\
\hline \multicolumn{5}{|l|}{ Gender } \\
\hline Boys & 320 & $61(19.1)$ & $1.38(0.83,2.27)$ & 0.209 \\
\hline Girls & 178 & $26(14.6)$ & 1 & \\
\hline \multicolumn{5}{|l|}{ Family size } \\
\hline > 7 members (large) & 215 & $41(19.1)$ & $1.21(0.76,1.93)$ & 0.412 \\
\hline$\leq 7$ members & 283 & $46(16.3)$ & 1 & \\
\hline \multicolumn{5}{|l|}{ Father's age (years) } \\
\hline$<40$ & 248 & $53(21.4)$ & $1.73(1.08,2.77)$ & $0.028^{*}$ \\
\hline$\geq 40$ & 250 & $34(13.6)$ & 1 & \\
\hline \multicolumn{5}{|l|}{ Residence } \\
\hline Almahweet (Highland) & 242 & $53(21.9)$ & $1.83(1.14,2.94)$ & $0.011^{*}$ \\
\hline Taiz (Coastal/foothill area) & 256 & $34(13.3)$ & 1 & \\
\hline \multicolumn{5}{|l|}{ Father's educational level } \\
\hline Non educated (< 6 years) & 281 & $52(18.5)$ & $1.18(0.74,1.89)$ & 0.489 \\
\hline Educated ( $\geq 6$ years) & 217 & $35(16.1)$ & 1 & \\
\hline \multicolumn{5}{|l|}{ Father's occupational status } \\
\hline Not working & 80 & $20(25.0)$ & $2.44(1.13,5.26)$ & $0.021^{*}$ \\
\hline Farmers and daily workers & 310 & $54(17.4)$ & $1.54(0.81,2.95)$ & 0.189 \\
\hline Government employees/professionals & 108 & $13(12.0)$ & 1 & \\
\hline \multicolumn{5}{|l|}{ Household monthly income } \\
\hline$<$ USD 80 (low) & 313 & $63(20.1)$ & $1.69(1.02,2.82)$ & $0.042^{*}$ \\
\hline$\geq$ USD 80 & 185 & $24(13.0)$ & 1 & \\
\hline \multicolumn{5}{|l|}{ Presence of improved toilet in house } \\
\hline No & 188 & $43(22.9)$ & $1.79(1.12,2.86)$ & $0.013^{*}$ \\
\hline Yes & 310 & $44(14.2)$ & 1 & \\
\hline \multicolumn{5}{|l|}{ Source of drinking water } \\
\hline Unimproved (well, streams, rain, dams) & 360 & $70(19.4)$ & $1.72(0.97,3.04)$ & 0.061 \\
\hline Improved (piped water) & 138 & $17(12.3)$ & 1 & \\
\hline \multicolumn{5}{|l|}{ Presence of domestic animals at household } \\
\hline Yes & 321 & $59(18.4)$ & $1.20(0.73,1.96)$ & 0.471 \\
\hline No & 177 & $28(15.8)$ & 1 & \\
\hline \multicolumn{5}{|c|}{ Presence of other family members infected with $H$. nana } \\
\hline Yes & 154 & $54(29.2)$ & $2.97(1.85,4.77)$ & $<0.001^{*}$ \\
\hline No & 344 & $42(12.2)$ & 1 & \\
\hline \multicolumn{5}{|l|}{ Washing hands before eating } \\
\hline No & 162 & $32(19.8)$ & $1.26(0.78,2.04)$ & 0.351 \\
\hline
\end{tabular}




\begin{tabular}{|c|c|c|c|c|}
\hline Yes & 336 & $55(16.4)$ & 1 & \\
\hline \multicolumn{5}{|c|}{ Washing hands after defecation } \\
\hline No & 144 & $34(23.6)$ & $1.76(1.08,2.85)$ & $0.021^{*}$ \\
\hline Yes & 354 & $53(15.0)$ & 1 & \\
\hline \multicolumn{5}{|c|}{ Washing hands after playing with soil } \\
\hline No & 199 & $44(22.1)$ & $1.69(1.06,2.69)$ & $0.026^{*}$ \\
\hline Yes & 299 & $43(14.4)$ & 1 & \\
\hline \multicolumn{5}{|c|}{ Indiscriminate defecation } \\
\hline Yes & 325 & $60(18.5)$ & $1.22(0.76,2.01)$ & 0.424 \\
\hline No & 173 & $27(15.6)$ & 1 & \\
\hline \multicolumn{5}{|c|}{ Cutting nails periodically } \\
\hline No & 278 & $52(18.7)$ & $1.22(0.76,1.95)$ & 0.414 \\
\hline Yes & 220 & $35(15.9)$ & 1 & \\
\hline \multicolumn{5}{|c|}{ Washing vegetables before eating } \\
\hline No & 152 & $37(24.3)$ & $1.91(1.18,3.07)$ & $0.008^{*}$ \\
\hline Yes & 346 & $50(14.5)$ & 1 & \\
\hline \multicolumn{5}{|c|}{ Washing fruits before eating } \\
\hline No & 133 & $31(23.3)$ & $1.68(1.03,2.74)$ & $0.038^{*}$ \\
\hline Yes & 365 & $56(15.3)$ & 1 & \\
\hline \multicolumn{5}{|c|}{ Boiling water before drinking } \\
\hline No & 454 & $81(17.8)$ & $1.38(0.56,3.36)$ & 0.483 \\
\hline Yes & 44 & $6(13.6)$ & 1 & \\
\hline
\end{tabular}

prevalence reported in Yemen and the Middle East region. Previous studies among primary schoolchildren in different governorates in Yemen reported prevalence rates between $2.3 \%$ and 13 \% (Raja'a et al., 2000; Azazy et al., 2002; Raja'a and Mubarak, 2006; Al-Haddad and Baswaid, 2010; Al-Mekhlafi et al., 2016; Alsubaie et al., 2016; Alwabr and Al-Moayed, 2016). The highest prevalence reported before the current study was $13 \%$ among children aged $5-18$ years in lbb governorate, which borders Taiz (Raja'a et al., 2000) followed by $12.2 \%$ reported among 320 children aged $6-16$ years in Hajjah governorate, which borders Almahweet (Alharbi et al., 2019). This variation in the prevalence of $H$. nana could be attributed to the different areas and populations studied as well as to the methods utilised for stool examination. A much lower prevalence rate $(0.9-1.4 \%)$ was reported among participants aged $2-80$ years attending hospitals and clinics in Sana'a City and Taiz governorate (Alyousefi et al., 2011; Al-Harazi, 2016). The higher prevalence reported by the current study may also be explained by the devastating ongoing civil war in Yemen that has led to approximately $55 \%$ of healthcare facilities being totally non-functioning (Gavlak, 2015; UNOCHA, 2019). Moreover, mass drug administration (MDA), the key strategy for controlling schistosomiasis and STH infections in Yemen, has been largely interrupted due to the war (Al-Mekhlafi et al., 2018).

A much lower prevalence of $H$. nana infection is commonly reported worldwide (Oliveira et al., 2015; Asai et al., 2016; Spinicci et al., 2018; Periago et al., 2018; von Huth et al., 2019). However, a similar prevalence (17.4\%) has been reported among children aged 3-16 years in the highlands of Peru (Cabada et al., 2016). In contrast, a higher prevalence rate has been reported in different countries around the world. For instance, $25.8 \%$ in 602 children aged 7 - 11 years in western Tajikistan (Matthys et al., 2011), $27.5 \%$ (195/708) in 708 children aged $8-16$ years in Nepal (Shrestha et al., 2018), $28.3 \%$ in 405 children aged less than 13 years in Ethiopia (Amare et al., 2013), $33 \%$ among 500 preschool children of displaced communities in Khartoum, Sudan (Abdel Hamid et al., 2015), $50 \%$ in 150 cases of phlyctenular eye disease in Egypt (Hussein and Nasr, 1991) and $55 \%$ among the aboriginal community in northwestern Australia (Reynoldson et al., 1997).

With regards to the risk factors of $H$. nana infection, the current study identified age below 6 years, presence of other family members infected with $H$. nana, highlands residency, living in a house without toilet facilities, not washing hands after defecation, and not washing vegetables before consumption as the significant risk factors. Interestingly, the current study revealed a significantly higher 
Table 4. Multivariate analysis of factors associated with $H$. nana infection among participants in rural Yemen $(n=498)$.

\begin{tabular}{lccc}
\hline Variables & \multicolumn{3}{c}{ H. nana infection } \\
\cline { 2 - 4 } & Adjusted OR & $95 \% \mathrm{Cl}$ & $P$-value \\
\hline Age (<6 years) & 4.28 & $2.04,8.98$ & $<0.001^{*}$ \\
Gender (males) & 1.32 & $0.76,2.29$ & 0.332 \\
Father's age (<40 years) & 1.63 & $0.98,2.55$ & 0.060 \\
Residence (highland) & 2.87 & $1.56,5.26$ & $0.002^{*}$ \\
Father's occupational status (not working) & 1.68 & $0.64,4.42$ & 0.289 \\
Father's occupational status (farmers and daily workers) & 0.94 & $0.40,2.23$ & 0.944 \\
Household monthly income (< USD 80) & 1.12 & $0.57,2.37$ & 0.679 \\
Presence of improved toilet in house (no) & 2.19 & $1.23,3.88$ & $0.008^{*}$ \\
Source of drinking water (unimproved) & 1.37 & $0.73,2.59$ & 0.325 \\
Presence of other family member infected with H. nana (yes) & 2.48 & $1.45,4.24$ & $0.001^{*}$ \\
Washing hands after defecation (no) & 1.88 & $1.08,3.27$ & $0.025^{*}$ \\
Washing hands after playing with soil (no) & 1.50 & $0.86,2.61$ & 0.149 \\
Washing vegetables before eating (no) & 2.11 & $1.06,4.19$ & $0.034^{*}$ \\
Washing fruits before eating (no) & 0.81 & $0.40,1.62$ & 0.549 \\
\hline
\end{tabular}

$\mathrm{OR}$, Odds ratio. $\mathrm{Cl}$, Confidence interval.

* Significant association (Adjusted $P<0.05$ ).

prevalence of $H$. nana infection among preschool-aged children (below 6 years) compared to older children. This is inconsistent with previous studies conducted in Zimbabwe (Mason and Patterson, 1994) and Peru (Cabada et al., 2016).

Currently, studies specifically among preschool children in Yemen are not available. However, a linear association between the prevalence of intestinal parasites and age is well documented. For instance, it is commonly reported that older children aged 10 years and above are more likely to contract infections from the environment (Cabada et al., 2016; Al-Mekhlafi et al., 2019). However, the nature and extent of the association might be different for some parasites, specifically $H$. nana and $E$. vermicularis because these can be transmitted directly from human to human and through auto-reinfection. The acquisition of $H$. nana infection can also be linked to the presence of other family members infected with $H$. nana, which the current study also identified as a risk factor of $H$. nana. This association might be attributed to the horizontal transmission (intrafamily transmission) of intestinal parasitic infections among family members. For instance, in poor communities an infected mother could transmit $H$. nana by preparing food for her family members, and infected children could also transmit the parasite to their siblings. These suppositions are consistent with previous studies on $\mathrm{H}$. nana and other intestinal parasites such as Giardia, Entamoeba and STH in Yemen and elsewhere (Mason and Patterson, 1994; Elyana et al., 2016; Al-Areeqi et al., 2017; Al-Mekhlafi, 2017)

The higher prevalence of $H$. nana among preschool children may have an important implication for the schistosomiasis and STH infections control programmes in Yemen and other countries that are endemic for Schistosoma and $H$. nana infections. These programmes cover only schoolchildren, whereas preschool-aged children and non-enrolled school-aged children, particularly girls, are not offered treatment (Oshish et al., 2011; Faust et al., 2020). In such circumstances, preschool children will continue serving as a source of infection within endemic communities, and other age groups will be reinfected within a short period of time. Therefore, it is vital to include preschool-aged children in MDA with praziquantel to improve the control of schistosomiasis and other intestinal parasitic infections (including $H$. nana) (Soares Magalhães et al., 2013; Asai et al., 2016). However, the absence of paediatric formulations of praziquantel hampers the routine treatment of preschool-aged children. Hence, the need for a paediatric formulation of praziquantel is pressing, and efforts are being undertaken to make it available by 2020 (Coulibaly et al., 2017; Fenwick, 2017). Indeed, the Pediatric Praziquantel Consortium, an international partnership, was established in 2012 with the goal of developing a pediatric-friendly praziquantel orally dispersible tablet formulation and obtaining regulatory approval of its use for treating schistosomiasis in children aged from 3 months to 6 years (https://www. pediatricpraziquantelconsortium.org/). Two paediatric clinical trials (Phase I and II) have been conducted in sub-Saharan Africa in the frame of the Consortium's project, and the Phase III trials of safety and efficacy of the developed formulation are ongoing (N'Goran et al., 2019; Faust et al., 2020).

In the current study, H. nana infection was found to be significantly higher among children residing in the highlands (Almahweet). These children had three times the odds of acquiring $H$. nana compared to their peers residing in coastal/foothill areas (Taiz). 
While the underlying reasons for such association remain unexplained, a similar topographical variation in $H$. nana prevalence has been reported in Peru where prevalence rates of $17.4 \%$ and $7.6 \%$ were found among children in coastal and highlands areas, respectively (Cabada et al., 2016; Vilchez Barreto et al., 2017). This suggests that further studies are needed on the impact of environmental and meteorological factors on the prevalence and distribution of $H$. nana.

It is also well documented that intestinal parasitic infections are particularly common in children living in underprivileged areas where poverty prevails and where sanitation and an improved water supply are lacking (Vaz Nery et al., 2019). In the current study, living in a house without functioning toilet facilities doubled the odds of children acquiring a $H$. nana infection. This is in agreement with previous studies in Mexico (Martinez-Barbabosa et al., 2010) and Peru (Vilchez Barreto et al., 2017). These two studies (Martinez-Barbabosa et al., 2010; Vilchez Barreto et al., 2017), as well as studies conducted in Kyrgyzstan (Steinmann et al., 2010) and Tajikistan (Matthys et al., 2011), have also identified the lack of an improved water supply in the household as a significant risk factor of $H$. nana; an association that was not reported in the current study.

Personal hygiene practices play a crucial role in the transmission of intestinal parasitic infections (Gungoren et al., 2007). The current study found that children who did not wash their hands after defecation and those who did not wash vegetables before consumption were approximately twice as likely to be infected with $H$. nana compared with children who practised good personal hygiene. This is consistent with previous reports on intestinal parasitic infections (Gulliver et al., 2014; Elyana et al., 2016; Al-Areeqi et al., 2017; Al-Mekhlafi, 2017). Thus, the findings of the current clearly speak to the imperative need for improved sanitation, water and hygiene (WASH) facilities in the rural communities of Yemen. Hymenolepis nana is the only cestode that does not necessarily require an intermediate host for its transmission from person to person. Infected individuals who do not wash their hands with soap after defecation may transmit infective eggs of $H$. nana to others by a variety of means including shaking hands and processing food. A previous randomised controlled trial showed that frequent handwashing with soap significantly decreased the $H$. nana reinfection rate among schoolchildren (Mahmud et al., 2015). Similarly, a systematic review of studies on the parasitic contamination of fruits and vegetables processed for human consumption showed that $H$. nana was among the most commonly reported parasites (Karshima et al., 2018).

Although $H$. nana infection is usually asymptomatic, it has been associated with gastrointestinal symptoms among children. The current study found a significant association between $H$. nana infection and abdominal pain and anal itching as well as dizziness. Although skin manifestations such as pruritus ani (anal itching) are rare in $H$. nana infection, a significant association between $H$. nana infection and anal itching was reported among the studied children. A previous study in Peru showed that $H$. nana infection is significantly associated with diarrhoea, jaundice, and headache (Cabada et al., 2016). Likewise, $H$. nana-infected Sudanese children have been found to have a nine-fold higher risk of diarrhoea compared with uninfected children (Abdel Hamid et al., 2015). Moreover, $H$. nana infection has been identified as a significant predictor of malnutrition and anaemia (Mohammad and Hegazi, 2007; Oliveira et al., 2015; Spinicci et al., 2018). Thus, further studies are warranted on the burden and clinical profile of $H$. nana infection among children in rural Yemen.

It should be noted that the current study has limitations that should be considered when interpreting the findings. Firstly, a cross-sectional study design was used for this study, which limited the ability to infer causality between $H$. nana infection and explanatory variables. Secondly, a single faecal sample was collected from each child instead of the ideal three consecutive days of sample, which would have enhanced the sensitivity of parasite diagnosis (Hall, 1981). Thus, the prevalence of $H$. nana infection reported in the current study might have been underestimated.

In conclusion, this study revealed a high prevalence of $H$. nana infection among children in rural Yemen, particularly among preschool-aged children. Therefore, preschool-aged children should be included in the national schistosomiasis and STH control programme. In addition to periodic MDA, the control programme should focus on providing health education on hygienic practices, the introduction of adequate WASH facilities and screening and treating other infected family members. These measures are crucial because they will help to significantly reduce the prevalence of $H$. nana and other intestinal parasites among rural communities in Yemen. Also, further investigations are required to evaluate the true public health burden and the clinical profile of $H$. nana infection in these communities in order to guide future control efforts against intestinal parasites.

\section{Acknowledgements}

The author would like to thank Dr. Abdulelah Al-Adhroey from Thamar University and Dr. Hany Sady from Hodeidah University, Yemen and Mr. Sadek Mahyoub and Mr. Haroon Shaddad for their fruitful cooperation during the field surveys and laboratory work. The author is also thankful to the children and their parents for their voluntary participation in this study. The work presented in this paper was funded by the University of Malaya Research Grant (RG331-15AFR).

\section{Conflict of interest}

The author states no conflict of interest.

\section{References}

Abdel Hamid, M.M., Eluack, I.A., Osman, M.K., ElaAgip, A.H., Muneer, 
M.S. (2015): The prevalence of Hymenolepis nana among preschool children of displacement communities in Khartoum state, Sudan: a cross-sectional study. Travel. Med. Infect. Dis., 13(2): 172 - 177. DOI: 10.1016/j.tmaid.2014.12.011

Al-Areegl, M.A., Sady, H., Al-Mekhlafi, H.M., Anuar, T.S., Al-Adhroey, A.H., Atroosh, W.M., Dawaki, S., Elyana, F.N., Nasr, N.A., ITHOI, I., LAU, Y.L., SURIN, J. (2017): First molecular epidemiology of Entamoeba histolytica, E. dispar and E. moshkovskii infections in Yemen: different species-specific associated risk factors. Trop. Med. Int. Health., 22(4): 493 - 504. DOI: 10.1111/tmi.12848 Alghazali, K.A., Teoh, B.T., Loong, S.K., Sam, S.S., Che-Mat-Serl, N.A., Samsudin, N.I., YaAcob, C.N., Azizan, N.S., Oo, A., Baharudin, N.A., Tan, K.K., Abd-JamlL, J., NoR'E, S.S., Khor, C.S., Joharl, J., MaHDY, M.A.K., AвUBAKAR, S. (2019): Dengue outbreak during ongoing civil war, Taiz, Yemen. Emerg. Infect. Dis., 25(7): 1397 - 1400. DOI: 10.3201/eid2507

AL-HADDAD, A.M., BASWAID, S.H. (2010): Frequency of intestinal parasitic infection among children in Hadhramout governorate (Yemen). J. Egypt. Soc. Parasitol., 40: 479 - 488

AL-HARAZI, T. (2016): Prevalence and risk factors associated with intestinal parasitic infection among patients in Taiz City, Yemen. Br. Microbiol. Res. J., 16(3): 28317

Alharbi, R.A., Alwajeeh, T.S., Assabrl, A.M., Almalki, S.S.R., AlruWETEl, A., AzAzY, A.A. (2019): Intestinal parasitoses and schistosome infections among students with special reference to praziquantel efficacy in patients with schistosomosis in Hajjah governorate, Yemen. Ann. Parasitol., 65(3): 217 - 223. DOI: 10.17420/ ap6503.203

Al-Mekhlafi, A.M., Abdul-Ghanl, R., Al-EryanI, S.M., SalF-Ali, R., MAHDY, M.A. (2016): School-based prevalence of intestinal parasitic infections and associated risk factors in rural communities of Sana'a, Yemen. Acta Trop., 163: 135 - 141. DOI: 10.1016/j.actatropica.2016.08.009

AL-MEKHLAFI, H.M. (2017): Giardia duodenalis infection among rural communities in Yemen: A community-based assessment of the prevalence and associated risk factors. Asian Pac. J. Trop. Med., 10(10): 987 - 995. DOI: 10.1016/j.apjtm.2017.09.011

AL-MEKHLAFI, H.M. (2018): Yemen in a time of cholera: current situation and challenges. Am. J. Trop. Med. Hyg., 98(6): 1558 - 1562. DOI: 10.4269/ajtmh.17-0811

Alsubale, A.S.R., Azazy, A.A., Omer, E.O.M., Al-Shibani, L.A., Al-MeKHLAFI, A.M.Q., AL-KhaWLANI, F.A. (2016): Pattern of parasitic infections as public health problem among school children: a comparative study between rural and urban areas. J. Taibah Univ. Med. Sci., 11: 91 - 178. DOl: https://doi.org/10.1016/j. jtumed.2015.10.006

AlwABR, G.M.A., AL-MoAyed, E.E. (2016): Prevalence of intestinal parasitic infections among school children of Al-Mahweet governorate, Yemen. Eur. J. Biol. Res., 6(2): 64 - 73

Alyousefi, N.A., MahdY, M.A., Mahmud, R., Lim, Y.A. (2011): Factors associated with high prevalence of intestinal protozoan infections among patients in Sana'a City, Yemen. PLoS One., 6(7): e22044.
DOI: 10.1371/journal.pone.0022044

Amare, B., Ali, J., Moges, B., Yismaw., G, Belyhun, Y., Gebretsadik, S., Woldeyohannes, D., Tafess, K., Abate, E., Endris, M., Tegabu, D., Mulu, A., Ota, F., Fantahun, B., Kassu, A. (2013): Nutritional status, intestinal parasite infection and allergy among school children in northwest Ethiopia. BMC Pediatr., 13: 7. DOI: 10.1186/14712431-13-7

Amer, A.S., SaAd, A.E., Antonios, S.N., Hasby, E.A. (2018): Prevalence of parasitic infections in surgically removed appendices: parasitological and histopathological studies. Helminthologia., 55(1): 33 - 44. DOI: 10.1515/helm-2017-0056

Asal, T., Córdova Vidal, C., Strauss, W., IKoma, T., Endoh, K., Yамамото, M. (2016): Effect of mass stool examination and mass treatment for decreasing intestinal helminth and protozoan infection rates in Bolivian children: a cross-sectional study. PLoS Negl. Trop. Dis., 10(12): e0005147. DOI: 10.1371/journal.pntd.0005147 Azazy, A.A., Al-MahbashI, T.Y., Al-MekHLafl, H.M. (2002): Prevalence of intestinal and blood parasites among school children in Sana'a and Al-Mahweet provinces, Yemen. Yemen Med. J., 4: $50-55$

Bendel, R.B., Afifl, A.A. (1977): Comparison of stopping rules in forward "stepwise" regression. J. Am. Stat. Assoc., 72: 46 - 53

Cabada, M.M., Morales, M.L., Lopez, M., Reynolds, S.T., Vilchez, E.C., Lescano, A.G., Gotuzzo, E., Garcia, H.H., White, A.C. JR. (2016): Hymenolepis nana impact among children in the highlands of Cusco, Peru: an emerging neglected parasite infection. Am. J. Trop. Med. Hyg., 95(5): 1031 - 1036. DOI: 10.4269/ajtmh.16-0237 Cheesbrough, M. (2005): District Laboratory Practice in Tropical Countries. Part 1. $2^{\text {nd }}$ edition, Cambridge, Cambridge University Press

Chero, J.C., Saito, M., Bustos, J.A., Blanco, E.M., Gonzalvez, G., GARCIA, H.H. (2007): Hymenolepis nana infection: symptoms and response to nitazoxanide in field conditions. Trans. R. Soc. Trop. Med. Hyg., 101: 203 - 205. DOI: 10.1016/j.trstmh.2006.04.004

Coulibaly, J.T., Panic, G., Silué, K.D., Kovač, J., Hattendorf, J., KEISER, J. (2017): Efficacy and safety of praziquantel in preschool-aged and school-aged children infected with Schistosoma mansoni: a randomised controlled, parallel-group, dose-ranging, phase 2 trial. Lancet Glob. Health., 5(7): e688 - e698. DOI: 10.1016/S2214-109X(17)30187-0

El Bcheraoui, C., Jumaan, A.O., Collison, M.L., Daoud, F., Mokdad, A.H. (2018): Health in Yemen: losing ground in war time. Global Health.,14(1): 42. DOI: 10.1186/s12992-018-0354-9

Elyana, F.N., Al-MekHlafi, H.M., Ithol, I., Abdulsalam, A.M., Dawaki, S., Nasr, N.A., Atroosh, W.M., Abd-Basher, M.H., Al-AreeQl, M.A., Sady, H., Subramaniam, L.R., Anuar, T.S., Lau, Y.L., Moktar, N., SuRIN, J. (2016): A tale of two communities: intestinal polyparasitism among Orang Asli and Malay communities in rural Terengganu, Malaysia. Parasit. Vectors., 9: 398. DOI: 10.1186/s13071016-1678-z

Faust, C.L., Osakunor, D.N.M., Downs, J.A., Kayun, S., Stothard, J.R., Lamberton, P.H.L., Reinhard-Rupp, J., Rollinson, D. (2020): 
Schistosomiasis control: leave no age group behind. Trends Parasitol., S1471-4922(20)30109-4. Retrieved May 22, 2020 from https:// www.sciencedirect.com/science/article/pii/S1471492220301094. DOI: 10.1016/j.pt.2020.04.012.

FENWICK, A. (2017): Schistosomiasis research and control since the retirement of Sir Patrick Manson in 1914. Trans. R. Soc. Trop. Med. Hyg., 111(5): 191 - 198. DOI: 10.1093/trstmh/trx036 Galan-Puchades, M.T. (2015): Hymenolepis nana vs. Taenia solium life cycle. Parasite Immunol., 37(8): 429. DOI: 10.1111/pim.12204 GaVLAK, D. (2015): Health system in Yemen close to collapse. Bull. World Health Organ., 93: 670 - 671. DOI: 10.2471/BLT.15.021015 Gulliver, F., Jeandron, A., Nguyen, V.A., Do, H.A., Ensink, J.H. (2014): Transmission of helminth eggs through hands in a highrisk community. Trans. R. Soc. Trop. Med. Hyg., 108: 670 - 672. DOI: 10.1093/trstmh/tru115

Gungoren, B., Latipov, R., Regallet, G., Musabaev, E. (2007): Effect of hygiene promotion on the risk of reinfection rate of intestinal parasites in children in rural Uzbekistan. Trans. R. Soc. Trop. Med. Hyg., 101(6): 564 - 569. DOI: 10.1016/j.trstmh.2007.02.011

HALL, A. (1981): Quantitative variability of nematode egg counts in faeces: a study among rural Kenyans. Trans. $R$. Soc. Trop. Med. Hyg., 1981;75:682 - 687. DOI: 10.1016/0035-9203(81)90148-6

HusseIN, A.A., NASR, M.E. (1991): The role of parasitic infection in the aetiology of phlyctenular eye disease. J. Egypt. Soc. Parasitol., 21(3): $865-868$

KARSHIMA, S.N. (2018): Parasites of importance for human health on edible fruits and vegetables in Nigeria: a systematic review and meta-analysis of published data. Pathog. Glob. Health., 112(1): 47 - 55. DOI: 10.1080/20477724.2018.1425604

KING, C.H. (2010): Cestodes (tapeworms). In: MANDELL, G.L., BENNETT, J.E., Dolin, R. (Eds) Mandel, Douglas and Bennett's Principles and Practice of Infectious Disease. $7^{\text {th }}$ edition, Philadelphia, Churchill Livingstone Elsevier

Lucas, S.B., Hassounah, O.A., Doenhoff, M., Muller, R. (1979): Aberrant form of Hymenolepis nana: possible opportunistic infection in immunosuppressed patients. Lancet., 2(8156 - 8157): 1372 - 1373. DOI: 10.1016/s0140-6736(79)92859-9

LWANGA, S.K., LemeSHow, S. (1991): Sample Size Determination in Health Studies: A Practical Manual. Geneva, World Health Organization

MACNISH, M. (2001): Characterization of community-derived Hymenolepis in Australia. PhD thesis, Australia, Perth: Murdoch University Medical Science. Retrieved February 20, 2020 from https:// researchrepository.murdoch.edu.au/id/eprint/176/2/02Whole.pdf Mahmud, M.A., Spigt, M., Bezabih, A.M., Pavon, I.L., Dinant, G.J., VELASCO, R.B. (2015): Efficacy of handwashing with soap and nail clipping on intestinal parasitic infections in school-aged children: a factorial cluster randomized controlled trial. PLoS Med., 12(6): e1001837. DOI: 10.1371/journal.pmed.1001837

Martinez-Barbabosa, I., Gutierrez-Cardenas, E., Gaona, E., Shea, M. (2010): The prevalence of Hymenolepis nana in schoolchildren in a bicultural community. Rev. Biomed., 21: e7
Mason, P.R., Patterson, B.A. (1994): Epidemiology of Hymenolepis nana infections in primary school children in urban and rural communities in Zimbabwe. J. Parasitol., 80(2): 245 - 250

Matthys, B., Bobieva, M., Karimova, G., Mengliboeva, Z., Jean-Richard, V., Holmnazarova, M., Kurbonova, M., Lohourignon, L.K., UtZINGER, J., WYSS, K. (2011): Prevalence and risk factors of helminths and intestinal protozoa infections among children from primary schools in western Tajikistan. Parasit. Vectors., 4: 195. DOI: 10.1186/1756-3305-4-195

MIRDHA, B.R., SamantRAY, J.C. (2002): Hymenolepis nana: a common cause of paediatric diarrhoea in urban slum dwellers in India. J. Trop. Pediatr., 48(6): 331 - 334. DOI: 10.1093/tropej/48.6.331 Mohammad, M.A., Hegazl, M.A. (2007): Intestinal permeability in Hymenolepis nana as reflected by non invasive lactulose/mannitol dual permeability test and its impaction on nutritional parameters of patients. J. Egypt. Soc. Parasitol., 37(3):877 - 891

Muehlenbachs, A., Bhatnagar, J., Agudelo, C.A., Hidron, A., Eberhard, M.L., Mathison, B.A., Frace, M.A., Ito, A., Metcalfe, M.G., Rollin, D.C., Visvesvara, G.S., Pham, C.D., Jones, T.L., Greer, P.W., Vélez Hoyos, A., Olson, P.D., Diazgranados, L.R., Zaki, S.R. (2015): Malignant transformation of Hymenolepis nana in a human host. N. Engl. J. Med., 373(19): 1845 - 1852. DOI: 10.1056/NEJMc1600490

National Information Centre (NIC) Yemen. (2019): Retrieved December 21, 2019 from http://www.yemen-nic.info/sectors/popul

N'goran, E., David Aka, N.A., Ouattara, M., Huber, E., Bezuidenhout, D., Kourany-Lefoll, E. (2019): Paediatric Praziquantel Consortium. Challenges and lessons from conducting a paediatric clinical trial in sub-Saharan Africa: The case of the praziquantel oral dispersible tablets phase II study in Côte d'Ivoire. Adv Parasitol., 103: 75 - 89. DOI: 10.1016/bs.apar.2018.09.002

O'DemPSEY, T. (2011): Helminthic infections. In: Finch, RG, Greenwood, D., Norrby, S.R., Whitley, R.J. (Eds) Antibiotic and Chemotherapy. $9^{\text {th }}$ edition, New York, Saunders Elsevier

Oliveira, D., Ferreira, F.S., Atouguia, J., Fortes, F., Guerra, A., Centeno-Lima, S. (2015): Infection by intestinal parasites, stunting and anemia in school-aged children from southern Angola. PLOS One., 10(9): e0137327. DOI: 10.1371/journal.pone.0137327

Olson, Pd, Yoder, K, Fajardo L-G, Lf, Marty, A.M., Van De Pas, S., Olivier, C., Relman, D.A. (2003): Lethal invasive cestodiasis in immunosuppressed patients. J. Infect. Dis., 187(12): 1962 - 1966. DOI: $10.1086 / 375357$

Oshish, A., Alkohlani, A., Hamed, A., Kamel, N., Alsoofi, A., Farouk, H., Ben-lsmall, R., Gabrielli, A.F., Fenwick, A., French, M.D. (2011): Towards nationwide control of schistosomiasis in Yemen: a pilot project to expand treatment to the whole community. Trans. $R$. Soc. Trop. Med. Hyg., 105(11): 617 - 627. DOI: 10.1016/j.trstmh.2011.07.013

OXFAM (2015): Two-thirds of people in conflict-hit Yemen without clean water. Retrieved December 22, 2019 from https://www.oxfam.org/en/pressroom/pressreleases/2015-05-26/two-thirds-people-conflict-hit-yemen-without-clean-water 
Periago, M.V., García, R., Astudillo, O.G., Cabrera, M., Abril, M.C. (2018): Prevalence of intestinal parasites and the absence of soil-transmitted helminths in Añatuya, Santiago del Estero, Argentina. Parasit. Vectors., 11(1): 638. DOI: 10.1186/s13071-0183232-7

Raja'A, Y.A., Assiragl, H.M., Abu-Luhom, A.A., Mohammed, A.B., AlBAHR, M.H., AsHAdDADI, M.A., AL MufLIHI, A.N. (2000): Schistosomes infection rate in relation to environmental factors in school children. Saudi Med. J., 21(7): 635 - 638

RAJA'A, Y.A., MuBARAK, J.S. (2006): Intestinal parasitosis and nutritional status in schoolchildren of Sahar district, Yemen. East Mediterr. Health J., 12: S189 - 194

Reynoldson, J.A., Behnke, J.M., Pallant, L.J., Macnish, M.G., Gilbert, F., GILES, S., SPARgo, R.J., THompson, R.C. (1997): Failure of pyrantel in treatment of human hookworm infections (Ancylostoma duodenale) in the Kimberley region of north west Australia. Acta Trop., 68(3): 301 - 312. DOI: 10.1016/s0001-706x(97)00106-x

SCHENONE, H. (1980): Praziquantel in the treatment of Hymenolepis nana infections in children. Am. J. Trop. Med. Hyg., 29: 320 - 321. DOI: 10.4269/ajtmh.1980.29.320

Shrestha, A., Schindler, C., Odermatt, P., Gerold, J., Erismann, S., Sharma, S., KoJu, R., Utzinger, J., Cissé, G. (2018): Intestinal parasite infections and associated risk factors among schoolchildren in Dolakha and Ramechhap districts, Nepal: a cross-sectional study. Parasit. Vectors., 11(1): 532. DOI: 10.1186/s13071-018-3105-0

Soares Magalhães, R.J., Fançony, C., Gamboa, D., Langa, A.J., SouSA-Figueiredo, J.C., Clements, A.C., Vaz Nery, S. (2013): Extending helminth control beyond STH and schistosomiasis: the case of human hymenolepiasis. PLoS Negl. Trop. Dis., 7(10): e2321. DOI: 10.1371/journal.pntd.0002321

Spiniccl, M., Macchioni, F., Gabriell, S., Rojo, D., Gamboa, H., Villagrán, A.L., Vallejos, Y., Strohmeyer, M., Roselli, M., Cancrin, G., Olliaro, P., Montresor, A., Bartoloni, A. (2018): Hymenolepis nana-an emerging intestinal parasite associated with anemia in school children from the Bolivian Chaco. Am. J. Trop. Med. Hyg., 99(6): 1598 - 1601. DOI: 10.4269/ajtmh.18-0397

Steinmann, P., Usubalieva, J., Imanalieva, C., Minbaeva, G., Stefiuk, K., JeAndRon, A., Utzinger, J. (2010): Rapid appraisal of human intestinal helminth infections among schoolchildren in Osh oblast, Kyrgyzstan. Acta Trop., 116: 178 - 184. DOI: 10.1016/j.actatropica.2010.06.008
THompson, R.C. (2015): Neglected zoonotic helminths: Hymenolepis nana, Echinococcus canadensis and Ancylostoma ceylanicum. Clin. Microbiol. Infect., 21(5): 426 - 432. DOI: 10.1016/j. cmi.2015.01.004

United Nations Office for the Coordination of Humanitarian Affairs, UNOCHA. (2019): Humanitarian response plan 2019. Retrieved February 10, 2020 from https://reliefweb.int/sites/reliefweb.int/ files/resources/2019_Yemen_HRP_V21.pdf

Vaz Nery, S., Pickering, A.J., Abate, E., Asmare, A., Barrett, L., Benjamin-Chung, J., Bundy, D.A.P., Clasen, T., Clements, A.C.A.., Colford, J.M. JR., Ercumen, A., Crowley, S., Cumming, O., Freeman, M.C., Haque, R., Mengistu, B., Oswald, W.E., Pullan, R.L., OlivelRA, R.G., Einterz OWen, K., Walson, J.L., Youya, A., Brooker, S.J. (2019): The role of water, sanitation and hygiene interventions in reducing soil-transmitted helminths: interpreting the evidence and identifying next steps. Parasit. Vectors., 12: 273. DOI: 10.1186/ s13071-019-3532-6

Vilchez Barreto, P.M., Gamboa, R., Santivañez, S., O'neal, S.E., Muro, C., Lescano, A.G., Moyano, L.M., Gonzalvez, G., Garcia, H.H., The Cysticercosis Working Group In Perú. (2017): Prevalence, age profile, and associated risk factors for Hymenolepis nana infection in a large population-based study in northern Peru. Am. J. Trop. Med. Hyg., 97(2): 583 - 586. DOI: 10.4269/ ajtmh.16-0939

Von Huth, S., Kofoed, P.E., Holmskov, U. (2019): Prevalence and potential risk factors for gastrointestinal parasitic infections in children in urban Bissau, Guinea-Bissau. Trans. R. Soc. Trop. Med. Hyg., 113: 545 - 554. DOI: 10.1093/trstmh/trz032

WHO/UNICEF. (2015): Progress on sanitation and drinking-water: 2015 update. World Health Organization and United Nations Children's Fund. Retrieved December 15, 2019 from http://files.unicef. org/publications/files/Progress_on_Sanitation_and_Drinking_Water_2015_Update_.pdf

WHO. (2006): Helminth control in school-age children A guide for managers of control programmes. Geneva, World Health Organization

Willcocks, B., McaulffFe, G.N., BaiRd, R.W. (2015): Dwarf tapeworm (Hymenolepis nana): characteristics in the Northern Territory 2002 - 2013. J. Paediatr. Child Health., 51(10): 982 - 987. DOI: 10.1111/jpc. 12885 